\title{
Philosophiques
}

\section{Alban Urbanas, La notion d'accident chez Aristote. Collection Noêsis, Bellarmin, Montréal, 1988.}

\section{Andrius Valevičius}

Volume 17, numéro 1, printemps 1990

URI : https://id.erudit.org/iderudit/027113ar

DOI : https://doi.org/10.7202/027113ar

Aller au sommaire du numéro

Éditeur(s)

Société de philosophie du Québec

ISSN

0316-2923 (imprimé)

1492-1391 (numérique)

Découvrir la revue

Citer ce compte rendu

Valevičius, A. (1990). Compte rendu de [Alban Urbanas, La notion d'accident chez Aristote. Collection Noêsis, Bellarmin, Montréal, 1988.] Philosophiques,

17(1), 159-160. https://doi.org/10.7202/027113ar d'utilisation que vous pouvez consulter en ligne.

https://apropos.erudit.org/fr/usagers/politique-dutilisation/ 
alban urbanas, La notion d'accident chez Aristote. Collection Noêsis, Bellarmin, Montréal, 1988.

\author{
par Andrius Valevičius
}

Urbanas nous donne une étude exhaustive, rigoureusement menée. En raison de l'absence d'une définition claire et nette d'accident chez Aristote, sa tâche n'a pas été facile. Il prend en considération une quantité imposante de littératures secondaires et démontre les «erreurs » de quelques interprétations " traditionnelles » comme celles de Brunschwig et Barnes.

L'A., dans la première partie de son livre, analyse la notion d'accident dans les « Topiques » et les « Analytiques ». À partir des « Topiques » il s'agit de déterminer la conception d'accident, soit positive, c'est-à-dire que l'accident correspond à un mode d'appartenance (que Urbanas a nommé le mode de la puissance) ou soit négative, ce qui ferait de l'accident quelque chose sans définition, sans propre ni genre, quelque chose qui appartient seulement à son sujet et qui peut aussi bien ne pas lui appartenir. La thèse d'Urbanas est d'affirmer la conception positive d'accident contre Brunschwig qui soutient la conception négative. Sous cette conception positive l'accident peut aussi ne pas appartenir, mais quand il appartient, cela lui donne une certaine nécessité :

En tant que mode particulier d'appartenance, l'accident est ce qui peut appartenir et ne pas appartenir ; mais en tant qu'il désigne un attribut appartenant réellement, l'accident est ce qui ne peut pas ne pas appartenir. Il en va de même d'un attribut appartenant réellement et selon le mode de la nécessité. (p. 42)

À partir des « Analytiques » I'A. considère la question des prédicables et la possibilité de les soumettre à une étude scientifique. Il arrive à une confrontation avec Barnes qui traduit "sumbebékos kath' autá " comme «accidents par soi » en entendant « accident», encore une fois, comme "ce 
qui n'est ni définition, ni genre, ni propriété » ou comme «ce qui peut appartenir et ne pas appartenir ». (p. 107) Or, la position d'Urbanas à la lumière de sa discussion des «Topiques» est de montrer que l'expression " accidents par soi » renferme une contradiction dans les termes : « En effet, ce qui est accidentel, c'est-à-dire contingent, c'est-à-dire qui peut appartenir et ne pas appartenir à un seul et même sujet, n'est jamais nécessaire ni essentiel ni, de ce fait, par soi ». (p. 126) Selon Urbanas, il faut traduire ce terme comme "prédicat par soi» ou par «attribut par soi», ce qui nous permet de désigner ce qui appartient à une chose plutôt comme une abstraction faite de son mode d'appartenance.

Dans la deuxième partie du livre l'A. traite de l'accident dans la Métaphysique. C'est ici que la position d'Aristote devient plus obscure. Urbanas nous montre que Aristore dans sa philosophie de l'être a finalement développé deux conceptions diamétralement opposées de l'accident. La première qui est négative, accorde aux accidents (sumbebékos) les caractéristiques suivantes:

(a) il est quelque chose du non être et n'a qu'une existence nominale ;

(b) il ne possède qu'une cause fortuite, indéterminée, accidentelle; et

(c) il ne fait l'objet d'aucune science. La seconde qui est positive, accorde aux accidents les trois caractéristiques suivantes: (a) il se détermine d'un mode d'être positif et jouit d'une existence objective ; (b) il vient à l'existence grâce à trois principes : la forme, le moteur, la matière ; et

(c) il fait l'objet d'une science abstraite. (p. 204)

Urbanas tente de montrer comment Aristote est conduit à adopter, en définitive, la seconde conception de l'accident; que la seconde conception est celle qui souligne encore une fois la primauté de la substance ou de la strucrure hiérarchique chez le Stagirite. Urbanas explique qu'avec la conception positive, l'accident est nécessaire sur le plan conceptuel, mais sur le plan ontologique il est non nécessaire.

Finalement, Urbanas veut proposer la thèse que la conception négative de l'accident n'a de sens que «dans une perspective de polémique où il s'agit pour Aristote de se démarquer des partisans du non-être ». (p. 204)

Un livre bien écrit, riche et instructif, mais parfois, justement à cause de l'épaisseur de son sujet, c'est un livre qui demande beaucoup d'efforts afin de suivre les longs arguments et épreuves logiques. Donc, c'est plutôt un livre pour les initiés que pour les laïcs en la matière.

Département de philosophie

Université de Montréal 